\title{
КОМБИНАТОРНЫЙ АНАЛИЗ СХЕМЫ РАЗМЕЩЕНИЯ РАЗЛИЧИМЫХ ЧАСТИЦ ПО РАЗЛИЧИМЫМ ЯЧЕЙКАМ БЕЗ ПУСТЫХ ЯЧЕЕК
}

\author{
Н. Ю. Энатская \\ Московский институт электроники и математики, \\ Национальный исследовательский университет «Высшая школа экономики», Россия
}

\begin{abstract}
Задачи анализа схемы здесь состоят в построении процедуры перечисления исходов схемы, определении их числа, решении задачи нумерации для этих исходов и их моделировании с использованием полученных здесь же результатов аналогичных исследований той же схемы без ограничений.
\end{abstract}

К лючев ы е с с о в а: различимые частицы; схема сочетаний; минимальные элементы в ячейках.

\section{N. Yu. Enatskaya. COMBINATORIAL ANALYSIS OF THE SCHEME OF ALLOCATION OF DISTINGUISHABLE PARTICLES INTO DISTINGUISHABLE CELLS WITHOUT EMPTY CELLS}

The problems of the scheme analysis here consist in arranging the procedure for enumerating the outcomes of the scheme, determining their number, solving the problem of numbering these outcomes, and their modelling using the results obtained here through similar studies of the same scheme but without restrictions.

Keywords: distinguishable particles; combination scheme; minimal elements in cells.

\section{ВВЕДЕНИЕ}

Пространство элементарных исходов нашей схемы состоит из части равновероятных исходов схемы размещений с повторением. Причем каждый исход нашей схемы является исходом общей схемы размещений с повторением, поэтому вероятность появления исходов нашей схемы среди исходов схемы размещений с повторением равна отношению чисел исходов нашей схемы к числу ее исходов. Распределение вероятностей исходов нашей схемы будем определять.

Вероятностный анализ общей схемы представляет собой известную «классическую задачу о дробинках», состоящую в нахождении вероятностного распределения числа пустых ячеек $\mu_{0}(r, n)$ при размещении $r$ различимых частиц по $n$ различимым ячейкам по схеме размещений с повторением [1].

Задачи комбинаторного анализа нашей схемы здесь состоят в построении процедуры перечисления исходов схемы, определении их числа, решении задачи нумерации (ЗН) для этих исходов и их моделировании с использованием полученных здесь же результатов аналогичных исследований той же общей схемы (без ограничения на отсутствие пустых ячеек).

Введем обозначения для заданных параметров схемы: $n$ - число ячеек, $r$ - число частиц. Число исходов нашей схемы $N_{A}$ выра- 
жается через числа Стирлинга второго рода $\sigma(r, n)$ по формуле $N_{A}=n ! \sigma(r, n)$.

В [1] в общей схеме получен закон распределения числа $\mu_{0}(r, n)$ пустых ячеек:

$P\left(\mu_{0}(r, n)=k\right)=C_{n}^{k}(1-k / n)^{r} P\left(\mu_{0}(r, n-k)=0\right)$,

где

$$
P\left(\mu_{0}(r, n)=0\right)=\sum_{i=0}^{n} C_{n}^{k}(-1)^{i}(1-i / n)^{r},
$$

откуда число исходов нашей схемы $N_{A}$ вычисляется по формуле

$$
N_{A}=n^{r} P\left(\mu_{0}(r, n)=0\right)=\sum_{i=0}^{n} C_{n}^{k}(-1)^{i}(n-i)^{r}
$$

найдены моменты для $\mu_{0}(r, n)$, представим, в частности, выражения для его математического ожидания - $M \mu_{0}(r, n)$ и дисперсии $D \mu_{0}(r, n)$ :

$$
\begin{gathered}
M \mu_{0}(r, n)=n(1-u / n)^{r} \\
D \mu_{0}(r, n)=n(n-1)(1-2 / n)^{r}+n(1-1 / n)^{r} \\
-n^{2}((1-1 / n))^{2 r} .
\end{gathered}
$$

Комбинаторный анализ нашей исследуемой схемы $A$ будет предварять проведение аналогичных исследований по направлениям перечисления и решения ЗН той же (общей) схемы без ограничений (схемы размещения с повторением - схема $B$ ), результаты которых будут использоваться. В ней при размещении $r$ различимых частиц по $n$ различимым ячейкам без ограничения числа частиц в ячейке известно число исходов $N_{B}=n^{r}$, а в перечислительной комбинаторике это одна из базовых схем. Естественная равновероятность исходов в ней соответствует равновероятности размещения частиц по ячейкам. Анализ схемы $B$, используемый здесь как вспомогательный, представляет отдельный интерес. В ней получено вероятностное распределение числа пустых ячеек из перечислительной процедуры ее исходов.

\section{1. АНАЛИЗ СХЕМЫ РАЗМЕЩЕНИЯ РАЗ- ЛИЧИМЫХ ЧАСТИЦ ПО РАЗЛИЧИМЫМ ЯЧЕЙКАМ - СХЕМЫ $B$}

\section{1. Вид исхода схемы и построение про- цесса их перечисления}

Перечисление исходов схемы будем производить методом графов (см. [6]) последовательным поединичным добавлением частиц от 1 до $r$ на каждом шаге их равновероятного размещения по $n$ ячейкам с графическим изображением этого процесса. Общее число исходов схемы на последнем $r$-м шаге должно быть равно $N_{B}$.

Исход $i$-го шага $(i=\overline{1, r})$ вида $R_{*}^{(i)}$ будем изображать $n$-мерным вектором $\left(s_{1_{i}}, \ldots, s_{n_{i}}\right)$, компоненты которого перечисляют через запятую номера элементов, попавших за $i$ шагов в подряд идущие ячейки с номерами от 1 до $n . \mathrm{B}$ графе каждый исход будем заключать в круглые скобки. Если в $j$-й ячейке $(j=\overline{1, n})$ на $i$-м шаге более одной частицы, то номера этих частиц в $j$-й компоненте исхода будем перечислять через запятую в возрастающем порядке и заключать в круглые скобки. Исходы на каждом шаге процесса их перечисления будем нумеровать в порядке роста номера ячейки добавления частицы на этом шаге. Обозначаем $N_{*}^{(r)}=N_{*}, R_{*}^{(r)}=R_{*}$.

Приведем пример перечисления исходов схемы (рис. 1).

Пример 1. Пусть $n=3, r=3$. Число исходов схемы $N=3^{3}=27$.

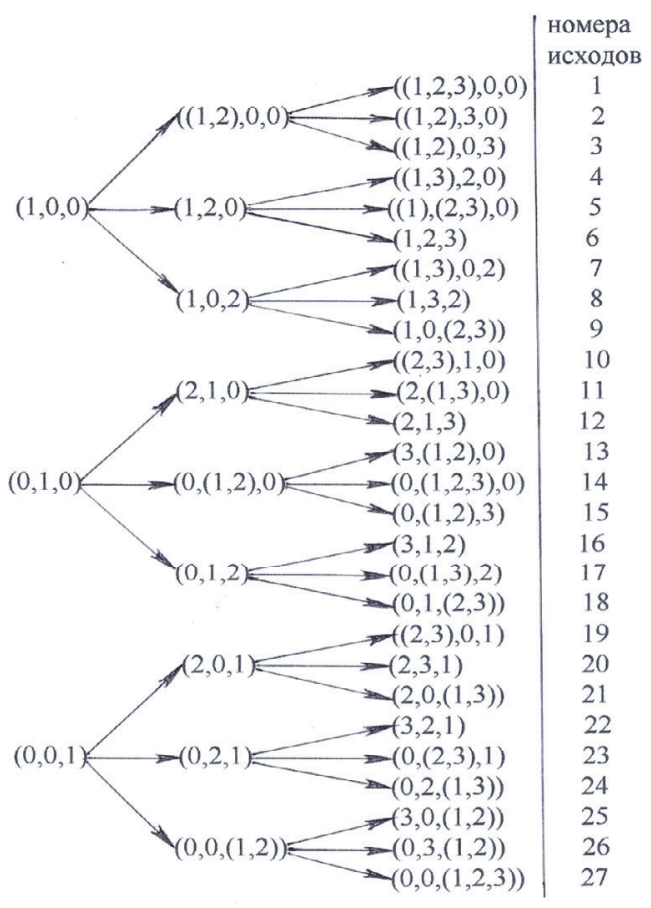

Puc. 1. Граф перечисления исходов схемы примеpa 1

Fig. 1. The enumeration graph of outcomes of scheme of example 1

\section{2. Задача нумерации (ЗН) исходов схе- мы}

Результаты прямой и обратной ЗН могут быть получены из [3] как частный случай оди- 
наковых чисел $n$ исходов каждого из последовательных действий. Приведем их в условиях и обозначениях исследуемой здесь схемы без ограничений в явном виде.

\section{Прямая ЗН}

Пусть дан номер исхода схемы $N_{*}=N_{*}^{(r)}$, требуется найти его вид $R_{*}$.

\section{Шаги решения:}

1) находим номера исходов траектории $T$ графа к конечному исходу с данным номером, т. е. находим $\bar{N}_{*}=\left(N_{*}^{(1)}, \ldots, N_{*}^{(r)}\right)$ рекуррентно, начиная с $N_{*}^{(r-1)}$ до $N_{*}^{(1)}$ по формуле

$$
N_{*}^{(i-1)}=\left[\frac{N_{*}^{i}+n-1}{n}\right]
$$

при $i=\overline{1, r}$, где $[Z]$ означает целую часть числа $Z$;

2) находим номера исходов траектории $T$ в пучках графа, т. е. находим $\bar{L}=\left(L_{1}, \ldots, L_{r}\right)$ рекуррентно по формуле

$$
L_{i}=N_{*}^{(i)} \bmod n+C_{n-v_{i}}^{n},
$$

где $v_{i}=N_{*}^{(i)} \bmod n$;

3) по результатам 2) выписываем искомый вид исхода, ставя каждое значение номера элемента $i$ в $R_{*}$ на место ее $L_{i}$-й компоненты.

Приведем числовой пример.

Пример 2. Пусть в условиях примера 1 дан номер исхода $N_{*}=N_{*}^{(3)}=13$. Требуется найти его вид $R_{*}$. По графу на рис. 1 $R_{*}=(3,(12), 0)$. Найдем теперь $R_{*}$ по алгоритму:

1) по (1) $N_{*}^{(2)}=[(13+3-1) / 3]=5 ; N_{*}^{(1)}=$ $[(5+3-1) / 3]=2$;

2) по $\left.(2) v_{1}=2 \bmod 3\right)=2 \Rightarrow C_{n-v_{1}}^{n}=0 \Rightarrow$ $L_{1}=2(\bmod 3)=2$,

$v_{2}=5 \bmod 3=2 \Rightarrow C_{n-v_{2}}^{n}=0 \Rightarrow L_{2}=$ $5 \bmod 3=2$,

$v_{3}=13 \bmod 3=1 \Rightarrow C_{n-v_{3}}^{n}=0 \Rightarrow L_{3}=$ $2 \bmod 3=1$;

3) из 2) в $R_{*} 1$ стоит во второй компоненте, 2 - во второй компоненте, 3 - в первой компоненте, откуда получаем $R_{*}=(3,(1,2), 0)$, что совпадает с результатом по графу.

\section{Обратная ЗН}

Пусть дан вид исхода схемы $R_{*}=R_{*}^{(r)}$, требуется найти его номер $R_{*}$.

\section{Шаги решения:}

1) исходя из того, что $L_{i}, i=\overline{1, r}$ - номер компоненты в $R_{*}$, содержащей номер элемента $i$, из данного $R_{*}$ получаем $\bar{L}=\left(L_{1}, \ldots, L_{r}\right)$;
2) находим номера исходов траектории $T$ по рекуррентной формуле при $\left(N_{*}^{(1)}=L_{1}\right)$

$$
N_{*}^{(i)}=\left(N_{*}^{(i-1)}-1\right) n+L_{i}
$$

при $i=\overline{2, r}$, откуда получаем искомый номер исхода

$$
N_{*}^{(r)}=\left(N_{*}^{(r-1)}-1\right) n+L_{r} \text { при } i=\overline{1, r} .
$$

Приведем числовой пример.

Пример 3. Пусть в условиях примера 1 дан вид исхода $R_{*}=R_{*}^{(3)}=(3,(1,2), 0)$. Требуется найти его номер $N_{*}$. По графу на рис. 1 $N_{*}=13$. Найдем теперь $N_{*}$ по алгоритму:

1) $\bar{L}=(2,2,1)-$ номера в пучках исходов траектории $T$, ведущей к исходу данного вида;

2) по (3) $N_{*}^{(1)}=2, N_{*}^{(2)}=(2-1) 3+2=$ $5, N_{*}^{(3)}=N_{*}=(5-1) 3+3=13$, что совпадает с результатом по графу.

\section{3. Моделирование исходов схемы $B$}

Для моделирования исхода схемы предлагается способ БМ, сложность которого будет определяться разыгрыванием случайного номера исхода схемы от 1 до $n^{r}$ при равновероятном распределении исходов и вычислением вида ее исхода по формулам (1) и (2) - результата решения прямой ЗН для нее, приводящий к линейной сложности.

\section{2. АНАЛИЗ СХЕМЫ РАЗМЕЩЕНИЯ РАЗ- ЛИЧИМЫХ ЧАСТИЦ ПО РАЗЛИЧИМЫМ ЯЧЕЙКАМ БЕЗ ПУСТЫХ ЯЧЕЕК - СХЕ- МЫ A}

Будем считать, что необходимое условие для возможной реализации схемы на ее параметры $r \geqslant n$ выполнено, частицы пронумерованы числами от 1 до $r$, а ячейки - от 1 до $n$. Под выбором частиц и ячеек подразумеваем выбор их номеров, а под сравнением элементов понимаем сравнение их номеров.

\section{1. Построение процесса перечисления исходов схемы и их число $N_{A}$}

Перечисление исходов схемы можно производить методом графов (см. [6]) путем отбраковки по данному ограничению исходов схемы $B$. Для численных расчетов при заданных конкретных значениях параметров схемы это, в силу простоты процедуры отбраковки, вполне приемлемый способ представления всех исходов нашей схемы $A$. Однако кроме численного подсчета числа $N_{A}$ исходов схемы такой подход связан с анализом лишних исходов и не дает общей формулы для $N_{A}$ как функции от ее параметров. Поэтому наряду с первым способом будем строить процесс прямого перечисления исходов схемы $A$. 
Прямое перечисление исходов схемы проводится в 3 этапа: 1-й состоит в фиксации всех составов минимальных номеров частиц в ячейках по схеме сочетаний [4] в возрастающем порядке; 2-й - в порционном добавлении остальных частиц в поединично растущие группы ячеек в порядке роста всех наборов фиксированных минимальных номеров в них на 1-м этапе, их сохраняющих по схеме размещения с повторением; 3-й - во всех перестановках, полученных на 2-м этапе заполнений всех ячеек. Здесь 1-й и 2-й этапы обеспечивают неповторяемость видов исходов схемы, а 3-й этап учитывает различимость ячеек.

\section{Шаги прямого перечисления.}

1) Из $r$ частиц выбираем всеми способами по схеме сочетаний $n$ частиц, перечисляем их в возрастающем порядке, в этом же порядке размещаем их по ячейкам с первой ячейки. Пусть это номера $\bar{r}=\left(r_{1}, \ldots, r_{n}\right)$.

2) Остальные $(r-n)$ частиц размещаем по всем ячейкам так, чтобы частицы, первоначально размещенные по одной в каждую ячейку в 1), были в них минимальными. Это значит, что в первой ячейке $r_{1}=1$ и обязательно находятся все частицы из остальных с номерами $<r_{2}$, в первых двух ячейках - равновероятно и обязательно все частицы из остальных с номерами $<r_{3}$, в первых трех - равновероятно и обязательно все частицы из остальных с номерами $<r_{4}$ и т. д., и, наконец, во всех ячейках - равновероятно все остальные частицы.

3) В каждом исходе 2) $n$ ! способами по схеме перестановок [5] переставляем составы $n$ ячеек. В результате получаем все исходы нашей схемы $A$.

По представленной логике прямого перебора исходов схемы $A$ найдем их число, используя операцию суммы по перечислению всех исходов 1) схемы сочетаний с числом исходов $C_{r-1}^{n-1}$ (без 1 , которая находится в первой ячейке в 1)) по всем описанным добавлениям остальных частиц, сохраняющим первые размещенные по ячейкам в 1) частицы минимальными при каждом фиксированном исходе пункта 1$): \bar{r}=\left(1, r_{2}, \ldots, r_{n}\right)$. Обозначим эту операцию по перечислению таких исходов в виде $\sum_{(C)}$ с перебором всех разных $\{\bar{r}\}$ и приведем формулу для $N_{A}$, отражающую перечисление исходов по всем описанным шагам:

$$
N_{A}=n ! \sum_{(C)}\left(\prod_{i=1}^{n-1} i^{r_{i+1}-r_{i}-1}\right) n^{r-r_{n}}
$$

Замечание 1. Здесь первый и второй шаги прямого перечисления исходов представляют перечисление исходов схемы (с теми же параметрами) размещения различимых частиц по неразличимым ячейкам без пустых ячеек.

Пример 4. В условиях примера 1 (рис. 1) по графу среди $3^{3}=27$ исходов схемы $B$ находим $N_{A}=6$ исходов схемы A: $\quad(1,2,3),(1,3,2),(2,1,3),(2,3,1),(3,1,2),(3,2,1)$. В операции по перечислению исходов схемы сочетаний - один исход $(1,2,3)$, откуда по (4) получаем $N_{A}=1^{2-1-1} 2^{3-2-1} 3$ ! $=6$, т. е. peзультаты совпали с визуальными по графу.

По приведенной во Введении формуле из [1] $N_{A}=C_{3}^{0}(-1)^{0} 3^{3}+C_{3}^{1}(-1)^{1} 2^{3}+C_{3}^{2}(-1)^{2} 1^{3}+$ $C_{3}^{3}(-1)^{3} 0^{3}=27-24+3-0=6$, что совпадает с вышеполученным результатом.

б) Для пояснения вычисления числа $N_{A}$ приведем еще пример с $n=3, r=4$ без графической иллюстрации, т. к. число исходов соответствующей схемы $B$ велико и равно $3^{4}=81$. Здесь по перечислению $(C)$, очевидно, имеем 3 исхода: $(1,2,3),(1,2,4),(1,3,4)$, откуда по (4) получаем $N_{A}=1^{2-1-1} 2^{3-2-1} 3^{4-3} 3 !+$ $1^{2-1-1} 2^{4-2-1} 3^{4-4} 3$ ! $+1^{3-1-1} 2^{4-3-1} 3^{4-4} 3$ ! = 3 ! $(3+2+1)=36$.

По приведенной во Введении формуле из [1] $N_{A}=C_{3}^{0}(-1)^{0} 3^{4}+C_{3}^{1}(-1)^{1} 2^{4}+C_{3}^{2}(-1)^{2} 1^{4}+$ $C_{3}^{3}(-1)^{3} 0^{4}=81-48+3-0=36$, что совпадает с вышеполученным результатом.

Замечание 2. Если по схеме сочетаний с повторением без пустых ячеек (с числом ячеек $n$ и числом частиц $r$ ) найти все наборы $\bar{m}^{(j)}=\left(m_{1}^{(j)}, \ldots, m_{n}^{(j)}\right), \quad j=\overline{1, C_{r-1}^{n-1}}$ уровней заполнения ячеек, то для числа исходов нашей схемы $N_{A}$ по схеме перестановок с повторением получаем новую формулу:

$$
N_{A}=\sum_{\left(C^{*}\right)} \frac{r !}{\prod_{i=1}^{n}\left(m_{i}^{(j)}\right) !},
$$

где сумма $\left(C^{*}\right)$ берется по всем наборам (группам) $\left\{\bar{m}^{(j)}\right\}$.

В условиях примера 4 в случаях а) $n=$ $3, r=3$ и б) $n=3, r=4$ вычислим по (5) число $N_{A}$ исходов схемы и сравним с полученными там значениями:

а) при $\left(C^{*}\right)=\{(1,1,1)\}$ получаем $N_{A}=$ $3 ! /(1 !)^{3}=6$;

б) при $\left(C^{*}\right)=\{(1,1,2),(1,2,1),(2,1,1)\}$ получаем $N_{A}=3 \cdot 4 ! / 2 !(1 !)^{2}=36$. Полученные результаты совпали с ранее полученными в примере 4 по формуле (4).

Визуально эти результаты легко проверяются:

а) здесь $\bar{m}^{(j)}=(1,1,1)$ - это по составу ячеек есть $(1,2,3)$, что при всех их перестановках дает $3 !=6$ исходов; 
б) здесь $\bar{m}^{(j)}=(1,1,2)$ - это по составу ячеек есть $(1,2,(3,4)),(1,3,(2,4)),(1,4,(2,3))$, $(2,3,(1,4)),(2,4,(1,3)),(3,4,(1,2))$, что при всех их перестановках дает по каждому $3 !=6$ вариантов, приводящих к их общему числу 36 исходов.

Замечание 3. Из замечания 1 следует, что из (4) $N_{A} / n$ ! есть число исходов схемы (с теми же параметрами) размещения различимых частиц по неразличимым ячейкам без пустых ячеек.

\section{2. ЗН в схеме $A$}

Схема $A$ представляет собой схему последовательных действий приведенных выше этапов прямого перечисления ее исходов, для которой ЗН решена в [3] при решенных ЗН всех трех ее этапов. Для 1-го и 3 -го этапов ЗН решены соответственно в [4] и [5]. Таким образом, решение $3 \mathrm{H}$ нашей схемы $A$ сводится к ее решению для 2-го этапа, т. е. для объединения действий схем $\left\{A_{j}\right\}$, состоящих в размещении $r$ различимых частиц по $n$ неразличимым ячейкам с $j$-м $n$-мерным, упорядоченным по возрастанию набором минимальных номеров содержащихся в них частиц, где под $j$ в схеме $A_{j}$ понимается $j$-й результат 1-го этапа прямого перечисления исходов схемы $A$ при $j=\overline{1, C_{r-1}^{n-1}}$. Решаем 3Н сначала в схеме $A_{j}$, а потом для 2-го этапа.

\subsection{1. ЗН в схеме $A_{j}$}

Схема $A_{j}$ представляет собой схему $n$ последовательных действий порционных добавлений остальных частиц в поединично растущие группы ячеек в порядке роста фиксированных минимальных номеров в них, их сохраняющих, а из [6] ЗН решена при известных размерах пучков этих $n$ действий, которые достаточно найти для решения здесь ЗН.

При фиксированном $\bar{r}$ приведем формулу для числа $N_{A_{j}}$ исходов схемы $A_{j}$ :

$$
N_{j}=N_{A_{j}}=n^{r-r_{n}} \prod_{m=1}^{n-1} m^{r_{m+1}-r_{m}-1} .
$$

Для наглядности приведем пример перечисления исходов схемы $A_{j}$.

Пример 5. Пусть $n=3, \bar{r}=(1,2,5)$, a) $r=5$; в) $r=6$.

Приведем граф перечисления исходов схемы с пошаговым порционным добавлением частиц в поединично растущие группы ячеек, описанного выше. В условиях небольших значений параметров легко проверить правильность пошагового перечисления исходов схемы и сравнить с вычисленным по (6).

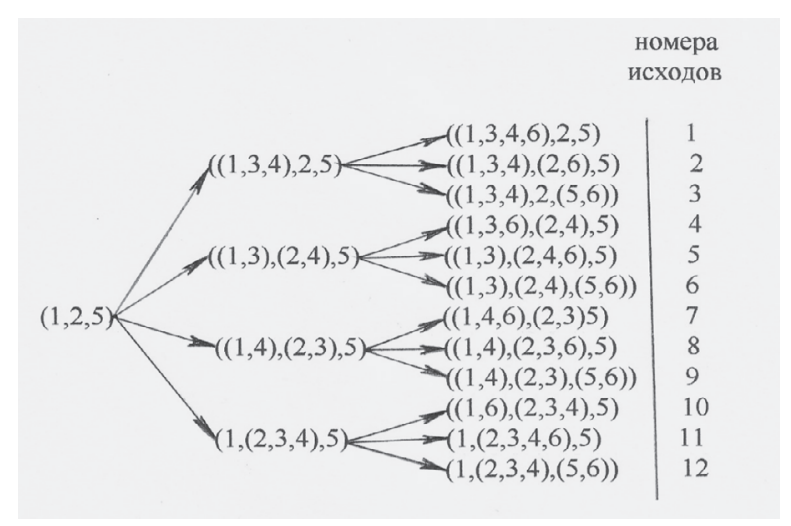

Puc. 2. Граф перечисления исходов схемы примеpa 5

Fig. 2. The enumeration graph of outcomes of scheme of example 5

Тогда по (6) имеем в случае а) $N_{A_{j}}=$ $2^{5-2-1} 3^{5-5}=4 ;$ а в случае в) $N_{A_{j}}=$ $2^{5-2-1} 3^{6-5}=12$, что совпадает с результатами чисел исходов итераций по графу на рис. 2 .

Пусть в схеме $A_{j}$ задан вектор $\bar{r}=$ $\left(1, r_{2}, \ldots, r_{n}\right)$. Тогда из логики прямого перечисления исходов схемы $A_{j}$ следует, что размер пучков $j$-го из $n$ последовательных действий схемы в графе их перечисления равен $i^{r_{i+1}-r_{i}-1}, i=\overline{2, n-1}$, при $i=1$ размер пучка есть $r_{2}-2$, а при $i=n$ размер пучков есть $n^{r-r_{n}}$. Таким образом, по [3] считаем решенной ЗН в этой схеме, т. е. что установлено взаимнооднозначное соответствие между номером ее исхода $N_{j *}$ и видом $R_{j *}$.

\subsection{2. ЗН 2-го этапа перечисления исхо- дов схемы $A$}

Для краткости обозначим схему этого пункта схемой $D$.

Схема $D$ представляет собой объединение $C_{r-1}^{n-1}$ действий по схеме $A_{j}$ по всем результатам первого этапа фиксаций векторов $\bar{r}$ с числами исходов из (6) $N_{j}, j=\overline{1, C_{r-1}^{n-1}}$ и решена в общем случае в [2].

Прямая ЗН. Пусть дан номер исхода $N_{*}^{(D)}$. Требуется найти его вид $R_{*}^{(D)}$.

\section{Шаги решения:}

1) находим номер $j$ схемы $A_{j}(j$-го пучка схемы $D)$, содержащий конечный с данным номером (искомый) исход в схеме $D$, по формуле

$$
j=\delta+\max t:\left(\sum_{l=1}^{t} N_{l}=S \leqslant N_{*}^{(D)}\right),
$$

где $\delta=0$ при $S=N_{*}^{(D)}$ и $\delta=1$ при $S<N_{*}^{(D)}$; 
2) находим номер $q$ искомого исхода в $j$-м пучке схемы $D$ :

$q=N_{*}^{(D)}-\sum_{l=1}^{t} N_{l}$

3) по номеру $q$ в $j$-м пучке схемы $D$, т. е. в $q$-м исходе схемы $A_{j}$, в которой $3 \mathrm{H}$ решена по результату прямой ЗН, находим искомый вид исхода $R_{*}^{(D)}$.

Обратная ЗН. Пусть дан вид исхода $R_{*}^{(D)}$. Требуется найти его номер $N_{*}^{(D)}$.

\section{Шаги решения:}

1) по виду исхода $R_{*}^{(D)}$ определяем номер $j$ исхода схемы сочетаний 1-го этапа перечисления исходов схемы $A$, т. е. номер пучка $j$ в схеме $D$;

2) по решенной обратной $3 \mathrm{H}$ в схеме $A_{j}$ по данному виду исхода $R_{*}^{(D)}$ находим его номер $q$ в схеме $A_{j}$, т. е. номер $q$ в $j$-м пучке схемы $D$;

3) по результатам 1) и 2) получаем $N_{*}^{(D)}=$ $\sum_{l=1}^{j-1} N_{l}+q$.

ЗН схемы $A$, представляющей собой схему последовательных действий трех этапов перечисления ее исходов, решена в [3] при решенных ЗН всех этапов, что мы теперь имеем в схеме $A$, т. к. для 3 -го этапа ЗН тоже решена в [5].

\section{3. Распределение вероятностей числа} пустых ячеек в общей схеме и распределение вероятностей исходов нашей схемы

Это распределение вероятностей получено в [1] и приведено во введении. Здесь оно будет представлено через $N_{A}=N_{A}(r, n)$.

По результату формулы (4) для $N_{A}(r, n)$ получаем вероятностное распределение для числа пустых ячеек $\mu_{0}$ в общей схеме, т. е.

$$
P\left(\mu_{0}=k\right)=\frac{C_{n}^{k} N_{A}(r, n-k)}{n^{r}},
$$

где $n^{r}$ в знаменателе - число исходов общей схемы, а в числителе стоит число исходов общей схемы с $k$ пустыми ячейками, получаемое $C_{r}^{k}$ вариантами выбора $k$ пустых ячеек и размещения в остальные $(n-k)$ ячеек $r$ частиц без пустых ячеек (при $r \geqslant n \quad k=\overline{1, n-1}$ ).

Вероятностное распределение исходов схемы $A$ будем находить удалением недопустимых состояний в графе схемы $B$ их перечисления по МГ, в котором исходы схемы равновероятны по п. 1.1. Все исходы схемы $B$ являются допустимыми в схеме $A$ при условии на число размещенных частиц $i:(r-i) \geqslant(n-1)$, означающем достаточность числа остальных ча- стиц для их размещения по всем пустым ячейкам при всех возможных вариантах после $i$-й итерации, т. е. до $i=i^{*}=r-n+1$ (включительно), после которой часть исходов схемы $B$ становятся недопустимыми для схемы $A$. Сформулируем ПРАВИЛА удаления недопустимых состояний с пропорциональным пересчетом вероятностей переходов в допустимые состояния схемы $A$ следующей итерации:

1) все состояния допустимы и не удаляются до $i^{*}$-й итерации включительно;

2) состояния недопустимы и удаляются на итерациях $i>i^{*}=r-n+1$, когда число пустых ячеек в них $>(r-i)$;

3) при числе пустых ячеек, равном $(r-i)$, на следующей итерации равновероятно размещаем частицу по всем пустым ячейкам;

4) удаление недопустимых состояний означает пропорциональное перераспределение вероятностей равновероятных переходов среди всех остальных состояний в пучке, содержащем это состояние.

Теперь приведем пояснительный пример вычисления вероятностного распределения исходов схемы $A$ путем их пересчета из равновероятных исходов схемы $B$.

Пример 6. Пусть $n=2, r=3$.

Приведем в данном примере граф перечисления исходов схемы $B$ (рис. 3 ).

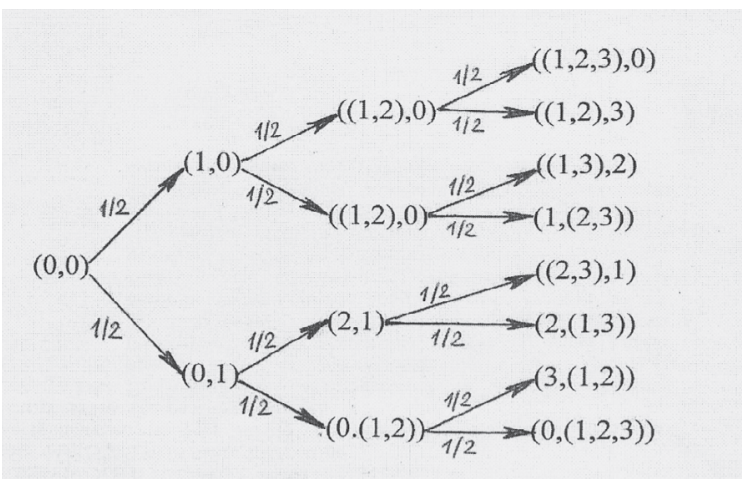

Puc. 3. Граф перечисления исходов схемы $B$ примера 6

Fig. 3. The enumeration graph of outcomes of scheme $B$ of example 6

Удаляя по одному крайнему недопустимому итоговому исходу с их траекториями и пропорциональным пересчетом итерационных вероятностей оставшихся исходов, получим граф перечисления исходов в схеме $A$ и по нему вычислим вероятности ее исходов:

$P((1,2), 3)=P(3,(1,2))=(1 / 2)^{2}=1 / 4$, a вероятности остальных четырех исходов по $(1 / 2)^{3}=1 / 8$. 
Проверка на распределение вероятностей: $4(1 / 8)+2(1 / 4)=1$.

\section{4. Моделирование исходов схемы $A$}

Оценим сложность способа моделирования одного исхода схемы $B$ через отбраковку исходов для схемы $A$ по отсутствию в ее исходе $R_{r}=\left(n_{1}, \ldots, n_{r}\right)$ хотя бы одного из номеров ячеек от 1 до $n$. Число таких исходов схемы $B$ до первого исхода схемы $A$, т. е. длина серии исходов схемы $B$, впервые заканчивающейся исходом схемы $A$, распределено по геометрическому закону с вероятностью исхода схемы $A$, полученного в п. 2.3 при $k=0$ по формуле для $P=P\left(\mu_{0}=0\right)$. Тогда с учетом этого сложность моделирования одного исхода схемы $A$ возрастает по сравнению со схемой $B$ в $1 / P$ раз, сохраняя линейную сложность.

\section{ЛИТЕРАТУРА}

1. Колчин В. Ф., Севаствянов Б. А., Чистяков В. П. Случайные размещения. М.: Наука, 1976. $223 \mathrm{c}$

\section{REFERENCES}

1. Kolchin V. F., Sevast'yanov B. A., Chistyakov $V$. P. Sluchainye razmeshcheniya [Random allocations]. Moscow: Nauka, 1976. 223 p.

2. Enatskaya N. $Y u$. Kombinatornyi analiz obobshchennoii skhemy posledovatel'nykh deistvii [Combinatorial analysis of the generalized scheme of the sequential actions]. Promyshlennye ASU $i$ kontrollery [Industrial ACS and Controllers]. 2016. No. 4. P. 25-27.

3. Enatskaya N. $Y u$. Kombinatornyi analiz skhem odnovremennykh i posledovatel'nykh deistvii [Combinatorial analysis of the schemes of simultaneous and sequential actions]. Promyshlennye ASU $i$ kontrollery [Industrial ACS and Controllers]. 2016. No. 2. P. 35-41.

\section{СВЕДЕНИЯ ОБ АВТОРЕ:}

\section{Энатская Наталия Юрьевна}

доцент Департамента прикладной

математики, к. ф.-м. н.

Национальный исследовательский университет

«Высшая школа экономики», Московский институт электроники и математики

ул. Таллинская, 34, Москва, Россия, 123458

эл. почта: nat1943@mail.ru

тел.: +79037411345
2. Энатская H. Ю. Комбинаторный анализ обобщенной схемы последовательных действий // Промышленные АСУ и контроллеры. 2016. № 4. C. $25-27$.

3. Энатская H. Ю. Комбинаторный анализ схем одновременных и последовательных действий // Промышленные АСУ и контроллеры. 2016. № 2. C. $35-41$.

4. Энатская Н. Ю. Комбинаторный анализ схемы сочетаний / / Промышленные АСУ и контроллеры. 2015. № 8. С. 33-38.

5. Энатская Н. Ю., Колчин А. В. Комбинаторный анализ схемы перестановок // Труды КарНЦ РАН. 2014. № 4. С. 80-86.

6. Энатская H. Ю., Хакимуллин Е. Р. Метод графов для решения задач перечислительной комбинаторики // Приборы и системы. Управление, контроль, диагностика. 2014. № 8. C. $15-21$.

Поступила в редакцию 09.01.2020

4. Enatskaya $N$. $Y u$. Kombinatornyi analiz skhemy sochetanii [Combinatorial analysis of combination scheme]. Promyshlennye ASU i kontrollery [Industrial ACS and Controllers]. 2015. No. 8. P. 33-38.

5. Enatskaya N. Yu., Kolchin A. V. Kombinatornyi analiz skhemy perestanovok [Combinatorial analysis of a permutation scheme]. Trudy KarNTs $R A N$ [Trans. KarRC RAS]. 2014. No. 4. P. 80-86.

6. Enatskaya N. Yu., Khakimullin E. R. Metod grafov dlya resheniya zadach perechislitel'noi kombinatoriki [Graphs method for solving enumerative combinatorics]. Pribory $i$ sistemy. Upravlenie, kontrol', diagnostika [Instruments and Systems. Management, Monitoring, and Diagnostics]. 2014. No. 8. P. 15-21.

Received January 09, 2020

\section{CONTRIBUTOR:}

Enatskaya, Natalia

National Research University

Higher School of Economics,

Moscow Institute of Electronics and Mathematics

34 Tallinskaya St., 123458 Moscow, Russia

e-mail: nat1943@mail.ru

tel.: +79037411345 